\title{
The viral anti-inflammatory chemokine-binding protein M-T7 reduces intimal hyperplasia after vascular injury
}

\author{
Liying Liu, ${ }^{1,2,3}$ Alshad Lalani, ${ }^{2,4}$ Erbin Dai, ${ }^{1,2,3}$ Bruce Seet, ${ }^{2}$ Colin Macauley, ${ }^{2,5}$ Raj Singh, ${ }^{2}$ \\ Lilly Fan, ${ }^{1}$ Grant McFadden, ${ }^{1,2}$ and Alexandra Lucas ${ }^{1,2,3}$
}

${ }^{1}$ Vascular Biology Research Group, Robarts Research Institute, and
2Department of Microbiology and Immunology, University of Western Ontario, London, Ontario, Canada
${ }^{3}$ Cardiovascular Research Group, Department of Medicine, Alberta Heritage Foundation Building, and
${ }^{4}$ Department of Biochemistry, University of Alberta, Edmonton, Alberta, Canada
${ }^{5}$ Viron Therapeutics Inc., London, Ontario, Canada

Address correspondence to: Alexandra Lucas, John P. Robarts Research Institute, Vascular Biology Research Group, University of Western Ontario, 100 Perth Drive, PO Box 5015, London, Ontario N6A-5K8, Canada. Phone: (519) 663-5777 ext. 34071; Fax: (519) 663-3789; E-mail: arl@rri.on.ca.

Received for publication November 17, 1999, and accepted in revised form April 25, 2000.

\begin{abstract}
Chemokines and IFN- $\gamma$ function as central regulators of inflammatory responses to vascular injury. Both classes of cytokines are upregulated during restenosis, a response to vascular injury that leads to recurrent atherosclerotic plaque growth, but the relative impact of each class of cytokines remains undetermined. M-T7 is a secreted myxoma viral immunomodulatory glycoprotein that functions both as a species-specific inhibitor of rabbit IFN- $\gamma$ and as a chemokine-binding protein, interacting with a wide range of C, C-C, and C-X-C chemokines in a species-nonspecific fashion. We wished to (a) assess the efficacy of purified M-T7 protein in inhibiting intimal hyperplasia after angioplasty injury and (b) exploit unique species-specific functions of M-T7 in order to judge the relative importance of each cytokine class on plaque growth. Anesthetized New Zealand white rabbits and Sprague-Dawley rats received either M-T7 or control at the time of arterial angioplasty injury. Histological analysis at 28 days demonstrated significant reductions in intimal hyperplasia with M-T7 treatment in both models, with an associated early inhibition of inflammatory cell invasion. Purified M-T7 protein inhibits intimal hyperplasia after angioplasty injury in a species-nonspecific fashion, thus implicating the chemokine-binding activity as more critical for prevention of plaque growth after vascular injury.
\end{abstract}

J. Clin. Invest. 105:1613-1621 (2000).

\section{Introduction}

Chemokines and IFN- $\gamma$ act as regulators of inflammatory reactions during early atherosclerotic plaque development (1-4). Both of these classes of cytokines have been detected in increased amounts at sites of vascular injury and atherosclerotic plaque development (5-12), but the relative roles of these inflammatory mediators in the accelerated atherogenesis after angioplasty (restenosis) remains undetermined.

IFN- $\gamma$ is released from activated $T$ cells, natural killer (NK) cells, and endothelial cells, possessing not only antiproliferative activities but also functioning as the major activator of macrophages in inflamed tissue (1-4, 13-18). Previous reports have associated administration of IFN with inhibition of smooth muscle cellular proliferation in tissue culture and reduced plaque growth in a hypercholesterolemic rabbit model $(19,20)$. IFN- $\gamma$ treatment of human monocytes was also found to reduce acetylated LDL uptake and to reduce intracellular cholesterol ester accumulation, inhibiting the formation of foam cells in tissue culture $(16,17)$. Other studies have, conversely, found that treatment with an antibody to IFN- $\gamma$ reduced plaque growth in a mouse heterotopic cardiac transplant model (21) and that the infusion of IFN- $\gamma$ induced arteriosclerotic arterial changes in pig- and human-to-immunodeficient SCID mouse xenografts (22). The function of IFN- $\gamma$ in atheroma development remains therefore unclear, with evidence both for proinflammatory and anti-inflammatory actions in animal models.

Chemokines are small 8-10-kDa proinflammatory proteins that act both as chemoattractants and activators of migrating leukocytes. The C-X-C class of chemokines classically is associated with the recruitment and activation of neutrophils whereas the C-C class of chemokines is targeted more to lymphocytes and macrophages, but the cellular specificities of the chemokine classes have subsequently been found to be less distinct (23-27). Mice deficient in monocyte chemoattractant protein-1 (MCP-1), and which also overexpress apo $\mathrm{B}$, were recently reported to have reduced plaque growth indicating that MCP-1 plays a role in plaque development (28). Knockout mice deficient in the CCR2 chemokine receptor (the receptor for MCP-1 and MCP-3) and crossed with atherogenic apo E-null mice were also demonstrated to exhibit reduced atherosclerotic plaque growth, further substantiating a central role for chemokines in early plaque development (29). Monocyte migration into areas of developing plaque has also been found to be closely associated 
with chemokine expression (30-32). Finally, an antimonocyte chemoattractant protein-1 (anti-MCP-1) antibody has also been reported to inhibit neointimal hyperplasia in injured rat carotid arteries (33).

Accelerated plaque growth after angioplasty, which can lead to restenosis, is characterized by an early inflammatory cell reaction with associated smooth muscle cellular proliferation and later connective tissue remodeling $(34,35)$. Restenosis is the major limiting factor in the long-term efficacy of percutaneous transluminal coronary balloon angioplasty (36), stent implantation (37), or other interventional therapies for occlusive coronary atherosclerosis (38). The initial complex cascade of biologic events that produces restenosis at previous angioplasty sites includes thrombosis, macrophage invasion, smooth muscle cellular migration, and proliferation and extracellular matrix production $(39,40)$.

The large DNA viruses, particularly the poxviruses and herpesviruses, have evolved numerous highly effective mechanisms designed to specifically shelter invading viruses from host immune and inflammatory responses that are intended to eradicate infecting viruses (41-44). Myxoma virus is a member of the poxvirus family that induces a highly virulent and lethal systemic disease in the European rabbit $(41,42)$. The virulence of myxoma virus infection in the mammalian host is mediated by viral immunomodulating factors that often target host inflammatory responses to infection (41-44). There is a direct interaction between viruses and effector cells that modifies the natural and acquired immune responses mediated through $\mathrm{T}$ and $\mathrm{B}$ lymphocytes, NK cells, monocytes, and macrophages $(43,44)$. Poxviruses encode a variety of gene products with significant homology to selected cellular cytokine receptors that are postulated to function by disturbing the cytokine network of infected hosts $(41-46)$. M-T7 is a secreted myxoma viral anti-inflammatory protein discovered to possess the dual functions of inhibiting IFN$\gamma$ ) by mimicking the IFN- $\gamma$ receptor (IFN $\gamma$-R) (45) and also acting as a chemokine-binding protein (46). The myxoma virus M-T7 protein bears significant homology to the ligand-binding domain of cellular IFN $\gamma$-Rs (45), but the IFN- $\gamma$ inhibitory activity is species specific, with binding exclusively limited to rabbit IFN- $\gamma(46)$. In contrast, M-T7 also binds a wide range of human, rabbit, and mouse $\mathrm{C}, \mathrm{C}-\mathrm{C}$, and $\mathrm{C}-\mathrm{X}-\mathrm{C}$ chemokines through the conserved $\mathrm{COOH}$-terminal heparin-binding domain (46) and has been postulated to function as a species pan-specific disrupter of chemokine gradients necessary for directed migration of inflammatory leukocytes into damaged or diseased tissues (46). Thus, in rabbits, M-T7 protein interacts with both IFN- $\gamma$ and chemokines, whereas in rat models, M-T7 possesses only the latter chemokine-binding activity.

We have used this differential species specificity of the myxoma M-T7 viral protein for these two contrasting anticytokine activities in order to assess the relative impact of inhibition of IFN- $\gamma$ and chemokines in animal models of atherosclerotic plaque growth after angioplasty balloon-mediated vascular injury. Plaque size at 28 days after injury, as well as early (24-hour) inflammatory cell invasion and cytokine expression, were assessed after infusion of purified M-T7 protein or controls in both rabbit and rat models of angioplasty balloon-mediated endothelial denudation.

\section{Methods}

Animal models. The effect of M-T7 treatment on cellular invasion and intimal hyperplasia was assessed in both rabbit and rat models of balloon angioplasty injury. M$\mathrm{T} 7$ was infused at the time of balloon-mediated arterial injury in 25 New Zealand white rabbits and in 52 Sprague-Dawley rats. The effects of M-T7 or control infusions were assessed by analysis of plaque growth at a 28-day follow-up. An additional 36 rats had early follow-up at 12 to 24 hours after M-T7 or control infusions given at the time of vascular injury.

Rabbit model of balloon injury. Twenty-five New Zealand white rabbits were fed $0.5 \%$ cholesterol in $6 \%$ peanut oil for 4 days per week, beginning 2 weeks before balloon injury and continuing for 28 days. A 3.0-3.5-mm angioplasty balloon catheter (1:1 diameter ratio of balloon to aorta) was inserted through a femoral arterial cut down and advanced retrograde to the abdominal aorta under general anesthetic (40 mg/kg ketamine (Vetrepharm Canada Inc., London, Ontario, Canada), $8 \mathrm{mg} / \mathrm{kg}$ xylazine, and $0.5 \mathrm{mg} / \mathrm{kg}$ acepromazine by intramuscular injection). The balloon was inflated to 8 atmospheres (atm) of pressure in the distal abdominal aorta, advanced to the level of the diaphragm, and withdrawn three times, under fluoroscopic guidance (47). Heparin (400 units, Leo Laboratories Canada Ltd.A; ax Ontario, Canada) was given immediately after obtaining femoral access. M-T7 was infused via a 3.0-3.5- $\mathrm{mm}$ diameter Wolinsky perforated balloon catheter (at an inflation pressure of $6 \mathrm{~atm}$ for 2 minutes) positioned in the abdominal aorta approximately $0.5 \mathrm{~cm}$ above the iliac bifurcation. M-T7 was infused at doses ranging from 50-5,000 pg as follows: saline $(n=6), \mathrm{M}-\mathrm{T} 750 \mathrm{pg}(0.017$ pg/g; $=6)$, M-T7 500 pg (0.17 pg/g; $n=6)$, M-T7 5,000 $\mathrm{pg}(1.7 \mathrm{pg} / \mathrm{g} ; n=7)$. Each infusate was administered as a diluted infusion in $0.9 \%$ saline, total volume of $10 \mathrm{~mL}$, immediately after balloon-mediated injury. To assess the effects of M-T7 on early cellular invasion, four rabbits had infusion of saline $(n=2)$ or $300 \mathrm{ng} \mathrm{M-T7}(n=2)$ immediately after angioplasty injury and were sacrificed 24 hours later for immunohistochemical analysis.

Rat model of balloon injury of the iliofemoral artery. To assess the effects of M-T7 on plaque growth after balloon injury and early inflammatory cell invasion, rats were divided into four groups, receiving either purified M-T7, heparin, a heparin/M-T7 mixture, or saline. Fifty-two male Sprague-Dawley rats (Charles River Laboratories, Wilmington, Massachusetts, USA) weighing 250-300 g were anesthetized with ketamine hydrochloride and Rompun 2:1 combination, $1 \mathrm{~mL} / 100 \mathrm{~g}$ weight intramuscular injection (Vetrepharm Canada Inc.). A $1.5-\mathrm{mm}$ diameter, 20-mm long angioplasty balloon catheter 

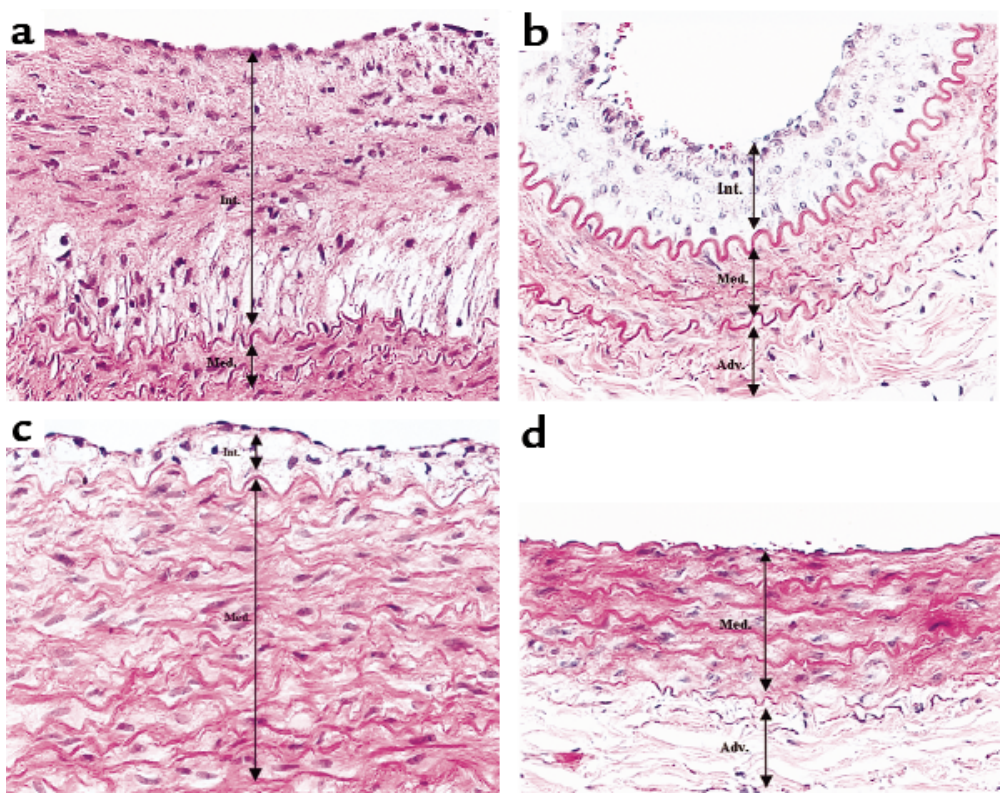

d

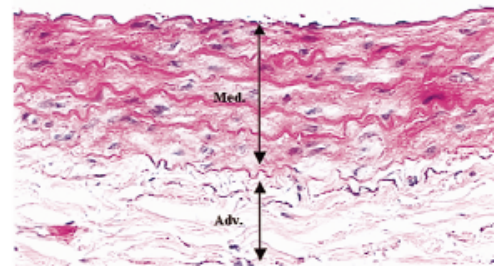

\section{Figure 1}

Hematoxylin and eosin-stained cross sections taken from rabbit abdominal aorta or rat iliofemoral artery after balloon injury at 28 days' follow-up $(\times 400)$. Moderately large areas of foam cell-filled, fatty plaque were seen in saline-treated rabbit aorta specimens (a), whereas less fatty areas of intimal hyperplasia with predominant smooth muscle cell invasion and proliferation were seen in the rat iliofemoral arterial specimens (b). M-T7 at $1.7 \mathrm{pg} / \mathrm{g}$ produced a marked reduction in plaque area in both the rabbit (c) and the rat models (d).
(Europass, Cordis Corp., Miami, Florida, USA) was introduced into the femoral artery using a cut-down procedure. All infusions $(1.0 \mathrm{~mL}$ volume of either M-T7 or controls diluted in $0.9 \%$ saline) were given immediately after balloon inflation through the distal lumen of the angioplasty balloon catheter into the arterial circulation, as described previously (48). The balloon was then inflated with saline to the point at which resistance to withdrawal was detected ( $6 \mathrm{~atm})$. The inflated balloon was then advanced and withdrawn from the iliac bifurcation to the distal femoral artery $(4-5 \mathrm{~mm})$ three times. The infusion doses were as follows: saline (0 pg M-T7; $n=16)$, M-T7 5 pg (0.017 pg/g; $n=6)$, M-T7 50 pg (0.17 pg/g; $n$ =6), M-T7 $500 \mathrm{pg}(1.7 \mathrm{pg} / \mathrm{g}, n=6)$, heparin $50 \mathrm{U}(n=12)$, heparin $50 \mathrm{U}$ mixed with M-T7 $500 \mathrm{pg}(1.7 \mathrm{pg} / \mathrm{g}, n=6)$. Heparin and the heparin/M-T7 mixtures were given by intravenous injection to assess the effects of heparin alone on plaque development and to assess potential interference by heparin on binding of M-T7 to the heparin-binding domain of chemokines. After the infusion, the catheter was removed and the femoral artery was sealed with local application of $n$-butyl cyanoacrylate monomer (Nexaband; Veterinary Products Laboratories, Phoenix, Arizona, USA).

To assess the early effects of M-T7 on inflammatory cell invasion, 36 rats had angioplasty injury of the iliofemoral artery (as just described) with immediate infusion of either saline control or 500 pg M-T7 under general anesthetic. Each group of rats was sacrificed at selected time intervals after balloon-mediated vascular injury and $M$ T7 or control infusion: at 0 hours $(n=8$ for saline, $n=6$ for M-T7), at 4 hours ( $n=8$ for saline, $n=4$ for M-T7), and at 24 hours ( $n=6$ for saline, $n=4$ for M-T7).

$M-T 7$ source and purification. M-T7 was isolated from baby green monkey kidney (BGMK) cells infected with a vaccinia vector expressing M-T7 and purified to apparent homogeneity by a two-step procedure using fast-performance liquid chromatography (FPLC), described in detail elsewhere $(45,49,50)$. Briefly, concentrated supernatants harvested from myxoma virus-infected BGMK cells were fractionated by anionexchange chromatography using a MonoQ HR5/5 column (Amersham Pharmacia Biotech Inc., Baie d'Urfé, Quebéc, Canada) and $\mathrm{NaCl}$ containing $20 \mathrm{mM}$ bis-Tris ( $\mathrm{pH}$ 6) elution buffer. MonoQ fractions containing $\mathrm{M}$ T7 were pooled, dialyzed against PBS overnight, and further purified by gel-filtration chromatography using a HiLoad Superdex 200 column (Amersham Pharmacia Biotech Inc.). Eluted proteins were subjected to SDS-12\% PAGE and visualized by silver staining and immunoblotting to assess the purity and composition of the purified proteins $(45,46,50)$. Fractions containing highly purified M-T7, were pooled, concentrated, and filter sterilized before they were injected.

Histological and morphometric analysis. At the designated follow-up times, study animals were sacrificed with Euthanyl (Bimda-MTC Animal Health Co., Cambridge, Ontario, Canada), and the arteries with previous angioplasty balloon-mediated injury were isolated for histological and morphometric analysis as described previously (47-49). Arterial sections $2.5 \mathrm{~cm}$ long from the rabbit aorta $(0.5-3.0 \mathrm{~cm})$ proximal to the iliac bifurcation and from the rat iliac artery bifurcation to the femoral arterial branches were harvested, divided into three sections $(0.8-1.0 \mathrm{~cm}$ long), and stained with hematoxylin and eosin for light microscopic and morphometric examination. Isolated arterial specimens were fixed in $10 \%$ sodium phosphate-buffered formalin, processed, impregnated, embedded in paraffin, and cut into $5-\mu \mathrm{m}$ sections by microtome, as described previously (47-49). Two sections were cut and stained from each of the three arterial sections from each animal specimen; a total of six sections were thus examined for each arterial specimen. 


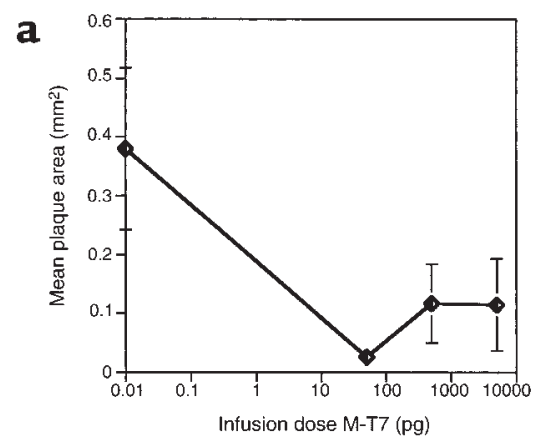

c

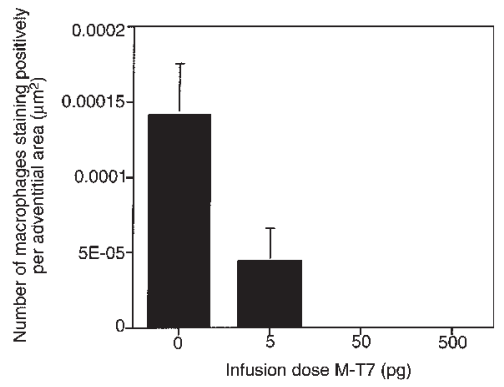

b

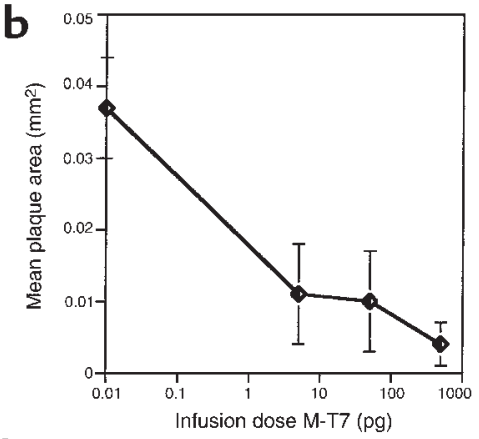

d

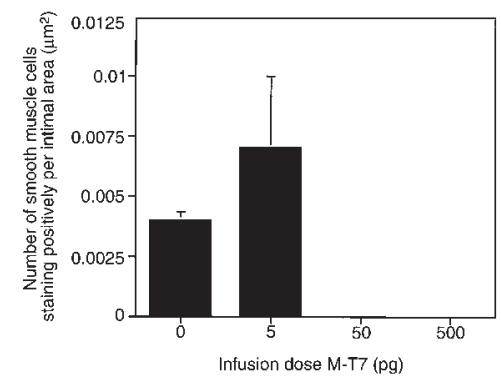

Figure 2

Line graphs demonstrate comparable reductions in plaque area expressed as mean plaque area $\left(\mathrm{mm}^{2}\right) \pm \mathrm{SE}$ in the rabbit model (a) and in the rat model of balloon injury (b) at 28 days' follow-up after angioplasty balloon injury. Bar graphs demonstrate dose-dependent reductions in invading macrophages in the adventitial layer (c) and smooth muscle cells in the intimal layer (d) expressed as mean number of cells staining positively per high-power field area $\left(\mu \mathrm{m}^{2}\right)$ at 28 days' follow-up.
Total intimal plaque area and the area of invading mononuclear cells were measured by morphometric analysis. Intimal plaque area was quantified using the section with the largest detectable area of atherosclerotic plaque by means of the Empix Northern Eclipse trace application program (Empix Imaging Inc., Mississauga, Ontario, Canada) using a Sony Power HAD $3 \mathrm{CCD}$ color video camera attached to the microscope and calibrated to the microscopic objective used. The mean total cross-sectional areas of the intima and the areas of inflammatory cell infiltration were then calculated for arterial specimens taken from the site of angioplasty balloon injury in each animal.

Immunobistochemical staining. For immunohistochemical analysis, formalin-fixed samples of arterial sections taken from the rat models (early and late follow-up) were embedded in paraffin blocks. The top and midarterial sections were used for immunohistological staining. After fixation, dehydration, and rehydration steps, the specimens were rinsed in PBS for 5 minutes. Tissue sections (5 $\mu \mathrm{m}$ thickness) were blocked with $20 \%$ normal horse serum for 10 minutes, then incubated with primary antibody for 1 hour to overnight, as determined previously (49), after which specimens were again rinsed in buffer. Sections were then incubated with primary antibodies specific to each cell type and selected cytokines. In all immunohistochemical staining, bound antibodies were detected using the indirect peroxidaselabeled antibody system (Vector Laboratories, Burlington, Ontario, Canada) as described previously (49). The primary antibody for arterial smooth muscle cells was mouse monoclonal IgG2 anti-alpha smooth muscle actin diluted 1:400 (Sigma Chemical Co., St. Louis, Missouri, USA). For macrophage staining, mouse monoclonal IgG anti-rat macrophage antibody (specific to ED2-like antigen on rat macrophage, not monocyte) at
1:100 dilution was used as the primary antibody (PharMingen, Mississauga, Ontario, Canada). For NK and maturing T-lymphocyte staining, mouse monoclonal anti-rat CD2 (IgG) thymocyte and T-lymphocyte antibody diluted 1:100 (Cedarlane Laboratories Ltd., Hornby, Ontario, Canada) was used as primary antibody (capable of recognizing maturing $\mathrm{T}$ lymphocytes and NK cells). For B-cell staining, mouse IgG anti-rat CD45RA (antibody to a subfraction of $240-\mathrm{kDa}$ rat CD45 specific to B lymphocytes) diluted 1:200 (Cedarlane Laboratories Ltd.) was used for the primary antibody (pan B cell). The primary antibody used for polymorphonuclear leukocytes (granulocytes) staining was mouse anti-rat IgM (1:100 dilution) with biotin-conjugated goat anti-mouse (rat adsorbed) Ig-specific polyclonal antibody (PharMingen). For the rabbit model, mouse RAM-11 anti-rabbit macrophage antibody (Cedarlane Laboratories Ltd.) was used for primary antibody for macrophage recognition. The following primary antibodies were used to assess chemokines present in the tissue specimens: rabbit anti-rat IgG for MCP-1, MIP-1 $\alpha$ (macrophage inflammatory protein$1 \alpha$ ), and RANTES (Cedarlane Laboratories Ltd.).

Treatment with the primary antibody was followed by incubation with biotinylated anti-mouse IgG diluted 1:250 (Vector Laboratories) for 20 minutes (for mouse primary antibodies) and goat anti-rabbit IgG (for rabbit primary antibodies) and avidin-biotin-peroxidase complex for 40 minutes. After rinsing, specimens were exposed to $3^{\prime} 3^{\prime}$-diaminobenzidine tetrahydrochloride (DAB) for 5 minutes. All specimens were counterstained with Harris hematoxylin solution for 5 minutes, then rinsed with distilled water, dehydrated, and clear mounted in cytoseal (Stephens Scientific Division of Cornwell Corp., Riverdale, New Jersey, USA) mounting medium for histologic assessment. 
The number of positively staining cells for the cellular analysis or the area staining positive for chemokines or cytokines was measured in three high-power fields, selected as having the largest area of positively stained cells or cytokine staining, from each slide and divided by the area of medial or adventitial layers of the artery wall examined. The final value for each arterial section was determined by the number of positively stained cells, or the area staining positive for chemokines and cytokines, and then divided by the area assessed for each of the three readings. A mean value was then calculated for each study animal. A negative control lacking primary antibody was present on each slide. Control stains lacking the secondary antibodies or using irrelevant primary antibody (IgG1 antibody to the cytoplasmic tail of vesicular stomatitis virus $G$ protein from $T$. kresi) were performed for each new antibody examined.

Statistical analysis. The mean values for plaque area or positively stained cells and chemokines were measured for all arterial specimens for each study animal. Correlations between the ratio of positively stained cells and measured plaque area with control or M-T7 treatment were determined by ANOVA and unpaired student's $t$ test. The results are summarized as mean value plus or minus SE, and significance was defined at $P$ less than 0.05 .

\section{Results}

Effect of M-T7 protein administration on neointima formation after vascular injury. In the control saline-treated rabbits, the $0.5 \%$ cholesterol/peanut oil diet, together with aortic injury, induced lipid-rich areas of plaque with increased macrophage invasion at the 28-day follow-up (Figure 1a). In contrast, at 28 days after balloon injury in rats fed a normal diet the plaque that developed in the iliofemoral artery was relatively rich in smooth muscle cells (Figure $1 \mathrm{~b}$ ). In both models, the foam cell rich-plaque in the cholesterol-fed balloon-injured rabbits (Figure 1c; $P$ < 0.0108 ) and the smooth muscle cell-rich intimal hyperplasia in the rat model (Figure $1 \mathrm{~d} ; P<0.0136$ ), there was a significant reduction in plaque growth with a single injection of M-T7 at the time of vascular balloon injury (Figures 1 and 2).

The reduction in intimal hyperplasia detected in both New Zealand white rabbits (Figure 2a) and SpragueDawley rats (Figure $2 \mathrm{~b}$ ) at the 28-day follow-up was comparable for both models at equivalent infusion doses. Relative to saline-treated control animals (rabbits or rats), an intra-arterial infusion of $0.017 \mathrm{pg} / \mathrm{g}$ or greater per rabbit (50 pg or greater total dose) and $0.017 \mathrm{pg} / \mathrm{g}$ or greater per rat (5 pg or greater total dose) given immediately after balloon angioplasty, produced the greatest reductions in neointima formation at the follow-up 28 days after M-T7 infusion. In the rabbit model, a relative increase in plaque area was noted at higher doses at 28 days after balloon injury, but this increase in intimal plaque size at higher infusion doses did not reach significance $(P=0.6504)$.
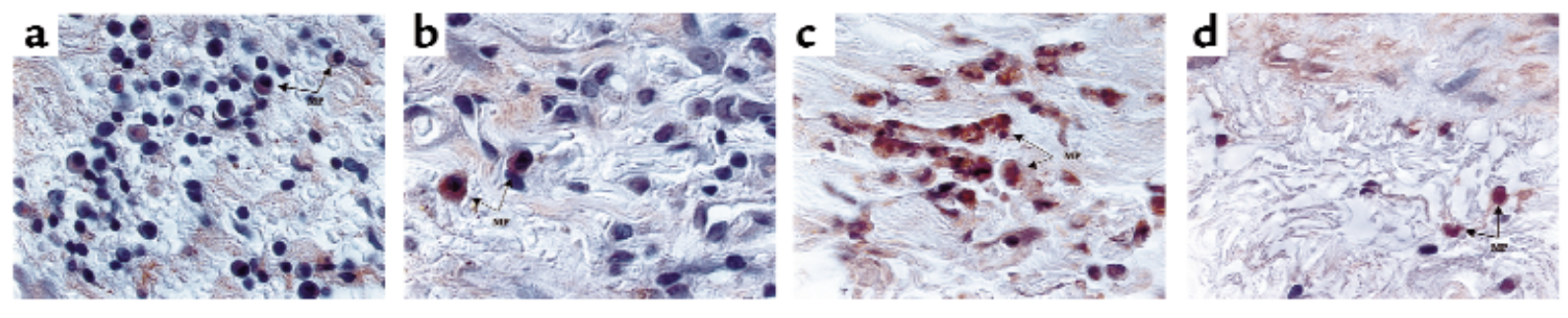

e

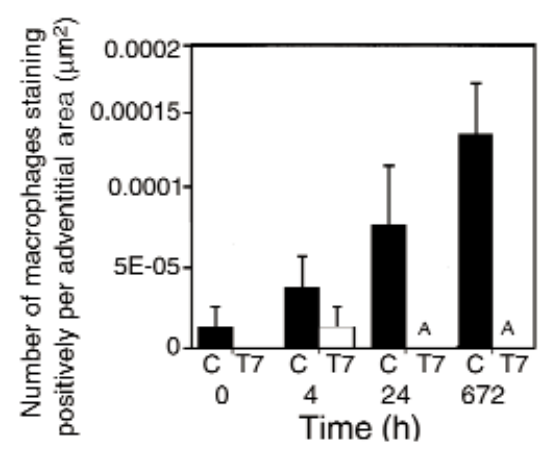

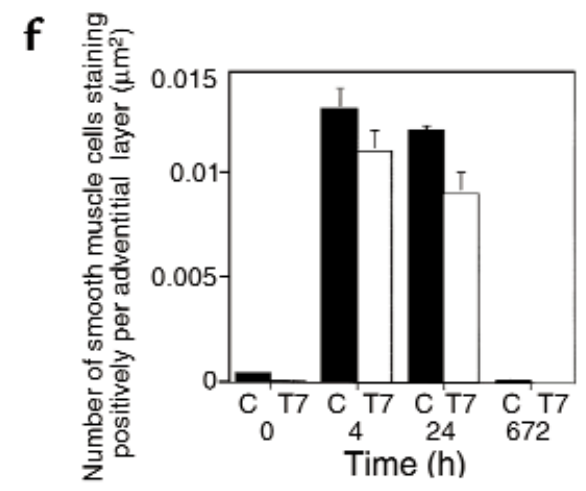

\section{Figure 3}

Immunohistochemical staining for late macrophage invasion (28 days) after balloon injury and saline control treatment in the rat iliofemoral artery (a). M-T7-treated sections $(1.7 \mathrm{pg} / \mathrm{g})$ of rat iliofemoral artery had a marked decrease in plaque area and macrophage invasion at 28 days' follow-up (b). An early increase in macrophage invasion into the medial and adventitial layers was detected at 24 hours' follow-up after angioplasty injury in saline-treated rat controls (c). M-T7 treated $(1.7 \mathrm{pg} / \mathrm{g})$ sections also demonstrated an early reduction in macrophage staining in the rat iliofemoral artery sections taken at 24 hours' follow-up on comparison with the control-treated rats (d). Bar graphs demonstrate decreased adventitial areas staining positive for macrophages $(\mathbf{e})$ and smooth muscle cells ( $\mathbf{f})$ in the rat balloon-injury model at early and late follow-up. C, saline control treated; T7, M-T7 treated. ×1,000. 
a
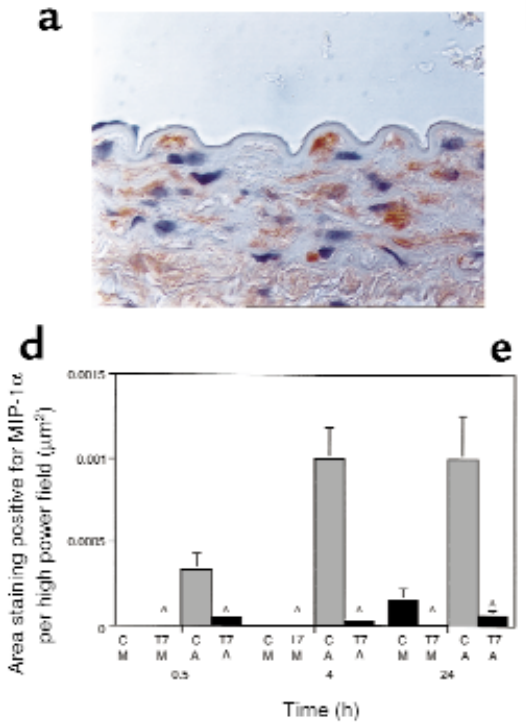

b
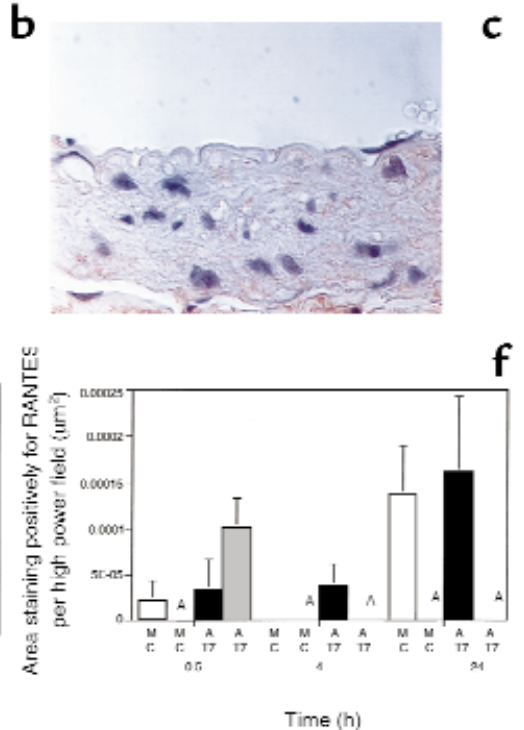

$f$
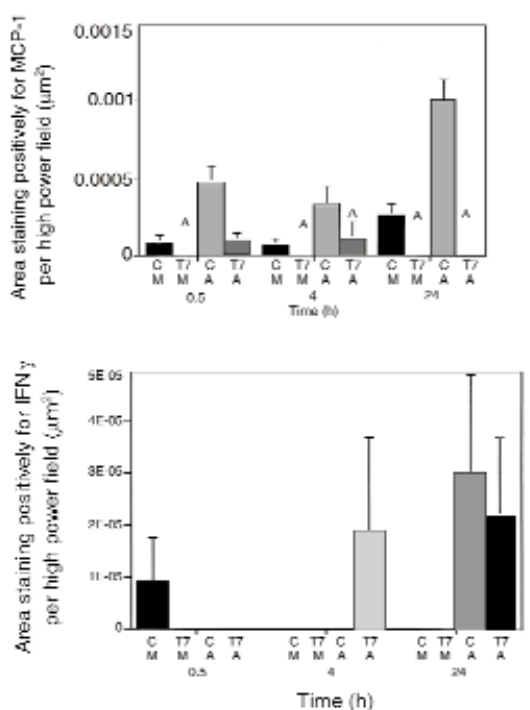

\section{Figure 4}

Immunohistochemical analysis of staining for MCP-1 in saline-treated rat arterial specimens (a) at 24 hours' follow-up after injury and in arterial sections taken from M-T7-treated specimens (b). Bar graphs demonstrate decreased detectable antigen for MCP-1 (c), MIP-1 $\alpha$ (d), and RANTES (e), but not IFN- $\gamma(\mathbf{f})$, after M-T7 treatment at 24 hours in arterial balloon-injury rat model at 24 hours' follow-up; expressed as the mean area staining positively per high-power field. M, media; A, adventitia, C, control saline treated; T7, M-T7 treated; solid bar, media control; right-slanted hatched bar, media M-T7 treated; cross-hatched bar, adventitia control; left-slanted hatched bar, adventitia M-T7 treated. ${ }^{A} P<0.01$.

M-T7-treated animals, in the rat or the rabbit model, had reduced mononuclear inflammatory cell invasion into both the intimal and the adventitial layers on comparison with saline-treated controls at the 28-day follow-up (Figures 2 and 3 ). The reduction in macrophage and smooth muscle cellular invasion in the intimal and adventitial layers was dose dependent (Figure 2c), with the greatest reduction seen at the two highest M-T7 infusion doses. Macrophage invasion was significantly reduced $(P<0.0011)$ even at the lowest dose of M-T7 (Figure 2c), whereas the smooth muscle cellular invasion was only significantly reduced at the highest M-T7 infusion dose $(P<0.0001)$ in the intimal layer (Figure $2 \mathrm{~d})$, indicating a dose-dependent effect of M-T7 on arterial cellular responses to vascular injury. Histological analysis and the cross-sectional area of the media did not differ significantly between the two groups after treatment with M-T7.

Immunohistochemical evaluation of cellular invasion. To evaluate the effects of M-T7 on cellular responses to vascular injury, sections of arteries were assessed by immunohistochemical analysis of individual cells, macrophages, smooth muscle cells, and CD-2-positive $T$ lymphocytes known to play a role in early and late atherosclerotic plaque development. The rat model was used initially in this study because of the known rabbit species-specific IFN- $\gamma$ binding activity of M-T7. The rat model was believed to be capable of reacting only to the species-nonspecific chemokinebinding activity of M-T7, which would allow the rat model to provide information on the specific effects of chemokine binding in early vascular responses to injury. The number of invading cells per high-power field in the intimal, medial, and adventitial layers were measured.

Invading macrophages were seen in arterial sections taken at the 28-day follow-up in the saline-treated rat model in all three arterial layers. The largest areas of macrophage invasion were seen in the intimal and the adventitial layers, with the greatest numbers of invading cells found in the adventitial layers. At 28 days' follow-up, these areas of macrophage invasion into the adventitial layer were markedly reduced after M-T7 infusion (Figure 3b) on comparison with control saline-treated rats $(P \leq 0.0016)$ (Figure 3a). A corresponding early reduction in macrophage invasion $(P \leq$ 0.0001 ) was detected in the adventitial layer of the arterial wall at 24 hours after balloon angioplasty in rats treated with M-T7 (Figure 3d) on comparison with control saline-treated animals (Figure 3c). There was no significant decrease in the numbers of invading macrophages in the adventitial layers at earlier times (at 0-4 hours) after injury with M-T7 treatment (Figure $3 e)$. Very few macrophages were detected in the medial arterial wall layers after angioplasty injury so that any potential effects of M-T7 on macrophage invasion into the media could not be accurately assessed. At 0-24 hours there was no intimal invasion; therefore, only data for cellular invasion from the medial and adventitial layers is discussed.

A trend toward a reduction in smooth muscle cell invasion was seen in the intimal and adventitial layers at the 28-day follow-up after M-T7 infusion (Figure 3f) on comparison with control saline-treated rat arterial 
specimens, but this did not reach significance $(P=$ $0.4659)$. Very few smooth muscle cells were detected migrating into the adventitial layer at early time points after balloon injury (0-24 hours after angioplasty injury) in these studies, thus preventing accurate assessment of effects of M-T7 on smooth muscle cellular invasion. Smooth muscle cellular count in the media was, however, unaffected by M-T7 treatment at early follow-up times ( $0-24$ hours; $P=0.3994)$, suggesting that no significant migration of smooth muscle cells out of the media was occurring at these early times after vessel injury.

Both CD2-positive T-lymphocyte and B-lymphocyte invasion were detected in very minimal amounts at early and late follow-up after balloon angioplasty injury. Neutrophils were not detected in these sections. In the adventitial layer the $\mathrm{B}$ cell and CD2-positive T-cell counts on immunohistochemical analysis were reduced at 28 days after M-T7 infusion (data not shown), but this reduction did not reach significance $(P=0.0966)$. The detected B-cell and CD2-positive T-cell numbers were, however, very small, even in the saline control animals, and analysis of effects of M-T7 on these cells was therefore not considered to be accurate.

For comparison, sections taken from the rabbit model at the 24-hour follow-up were also assessed for the effects of M-T7 infusion on macrophage and smooth muscle cellular invasion. The rabbit aortic histological sections had a similar decrease in the numbers of invading macrophage cells at 24 hours' follow-up after treatment with M-T7. Both the medial and adventitial layers had reductions in cells staining positive for RAM-11 (macrophages). In the abdominal aortic sections the mean number of macrophages per square micrometer was $0.0003 \pm 0.0003$ for saline-treated rabbit aorta and $0.0000061 \pm 0.0000061$ for M-T7-treated rabbit aorta in the medial layers $(P<0.0363)$ and $0.000033 \pm$ 0.000061 for saline-treated rabbits and $0.0 \pm 0.0$ for the adventitial layers $(P=0.21)$. There was no significant change detected in the number of smooth muscle cells at 24 hours, as seen in the rat model (data not shown).

Immunohistochemical analysis of IFN- $\gamma$ and chemokine antigen expression in injured iliofemoral arteries. Because M-T7 is known to function in vitro both as an IFN $\gamma$ - $\mathrm{R}$ homologue and chemokine-binding protein, we have used immunohistochemical staining to assess detectable IFN- $\gamma$ and chemokine (specifically MCP- 1, MIP- $1 \alpha$, and RANTES) antigens in the arterial wall after angioplasty injury and M-T7 infusion in the rat model. There was no change in MCP- $1 \alpha$, MIP- $1 \alpha$, and RANTES at 0 and 4 hours, but there was a significant reduction in the measured areas in the medial layer staining positively for MIP-1 $\alpha(P \leq 0.0001)$, MCP-1 $(P=0.0147)$, and RANTES $(P \leq 0.0001)$ (in the medial layer 24 hours after M-T7 treatment, as demonstrated for MCP-1 in Figure 4 , $a$ and $b$. Only MIP- $1 \alpha$ was significantly reduced after M-T7 treatment in the advential layers $(P \leq 0.0008)$ at 24-hours' follow-up. A bar graph comparing the mean ratios of positively stained areas for IFN- $\gamma$ and the chemokines tested per high-power fields demonstrates this relative decrease in early detectable chemokine antigen expression after M-T7 treatment on comparison with saline-infused control arteries (Figure 4, c-f). Selective immunohistochemical staining for IFN- $\gamma$ did not show a significant decrease in vascular IFN- $\gamma$ after M-T7 treatment when compared with the saline-treated controls at any of the times tested in either the medial or the adventitial arterial layers in the rat angioplasty injury model (Figure 4f; $P=0.3030$ ).

Effect of heparin on intimal hyperplasia during $M-T 7$ infusion. As M-T7 binds to the heparin-binding domain of the chemokine molecule (45), potential effects of heparin, (either direct or indirect through chemokine binding) on M-T7 activity during early plaque development was assessed after infusion of heparin, heparin mixed with M-T7, M-T7 alone, and saline immediately after angioplasty injury in our rat model. The results show that only when M-T7 alone was present was a significant reduction in intimal hyperplasia detected on comparison with saline or heparin infusions (M-T7, $0.006 \mathrm{~mm}^{2} \pm 0.004 ; \mathrm{M}-\mathrm{T} 7+$ heparin, $0.018 \mathrm{~mm}^{2} \pm$ 0.007; heparin, $0.025 \mathrm{~mm}^{2} \pm 0.007$; saline, $0.037 \mathrm{~mm}^{2} \pm$ 0.007). As noted previously, M-T7 treatment reduced plaque growth significantly when compared with saline infusion $(P \leq 0.0209)$, but the reduction in plaque size after treatment with M-T7 combined with heparin $(P=$ $0.1319)$ or heparin alone $(P=0.2813)$ was not large enough to reach significance. The presence of heparin thus did interfere with M-T7 antiatherogenic activity in the rat model, although a nonsignificant trend toward a reduction in intimal area was detected on comparison with heparin or saline treatments. M-T7 reduced plaque growth to the same degree as when infused alone. This may be due to an independent activity of heparin or due to competition of heparin with M-T7 for chemokine binding.

\section{Discussion}

We have demonstrated that the myxoma virus protein M-T7, with the dual functions of species-specific IFN$\gamma$ and species-nonspecific chemokine-binding activity, inhibits plaque development with similar efficacy in both rabbit and rat models of angioplasty-mediated vascular injury and with similar ranges of effective dose response. We postulate that the broad-based species-nonspecific chemokine-binding activity of $M$ $\mathrm{T} 7$ is responsible for the reduction in plaque growth in the rat and the rabbit models. The IFN $\gamma$-R homologue activity is rabbit species specific and hence cannot function in the rat model. This IFN- $\gamma$ binding activity would also be predicted to increase plaque growth based upon the previous observations of reduced plaque growth seen in cholesterol-fed rabbits treated with interferon $(19,20)$. The reduction of plaque in both models after a single infusion of M-T7 immediately after vascular injury therefore suggests the following: (a) that chemokines play a critical early role in activation of the inflammatory response in vascular 
wound healing and (b) the extent of M-T7 antiatheroma activity measured cannot be attributed to the inhibition of IFN- $\gamma$ in this rat model.

The attraction of inflammatory cells to damaged or infected tissues is essential for the host responses to injury and infection. The inflammatory cell response process is controlled by chemokines, chemotactic cytokines (22-26), peptides that are also believed to have central roles in early events leading to plaque growth at sites of vascular injury (27-29). The importance of chemokines in the pathophysiologic process of disease is now understood, but the role of chemokines in recurrent plaque growth after angioplasty is still being defined. Further, the relative effects of chemokine and IFN- $\gamma$ inhibition on later intimal hyperplasia produced by angioplasty injury remains to be determined. We have tested for local early arterial expression of three C-C chemokines, specifically MIP-1 $\alpha$, MCP- 1 , and RANTES, as well as rat IFN- $\gamma$, in the rat iliofemoral artery injury model. The rat model alone was tested to assess the effects of the isolated chemokine-binding activity of MT7 on early expression of chemokines after vascular balloon-mediated injury. IFN- $\gamma$ expression, although not predicted to be altered by M-T7 treatment in the rat model of balloon injury, was also tested as a control. We detected a significant reduction in chemokine antigens (Figure 4, c-e), but not IFN- $\gamma$ (Figure 4f), using immunohistochemical analysis of arterial specimens taken at 0 , 4 , and 24 hours after angioplasty. This reduction in chemokine staining may be the result of a primary alteration in local tissue levels of chemokines by M-T7. The reduction in chemokine staining at early times after balloon injury in the presence of M-T7 may also be the product of an inhibition of chemokine mRNA or protein expression, or due to interference with antibody binding to chemokines already present in the vascular wall during immunohistochemical staining, or potentially even due to a downstream effect of an as yet unknown function of M-T7 on the inflammatory system response. The fact that IFN- $\gamma$ antigen expression was not altered after M-T7 treatment in rats, however, would suggest that this observed reduction in plaque growth is not due to a generalized downregulation of inflammatory cytokine responses. It must be noted, however, that the amount of IFN- $\gamma$ detected was small as seen by immunohistochemical analysis and thus accurate detection of a reduction of IFN- $\gamma$ by this method is difficult and should be investigated further. Future experiments, perhaps with M-T7 variants lacking chemokine-binding activity, will need to be done to assess the definitive mechanism(s) of action of M-T7 in the prevention of injury-induced plaque growth. This study does, however, demonstrate that the protective antiatherogenic state after arterial trauma in these two models of angioplasty injury by M-T7 cannot be explained by the species-specific inhibition of IFN- $\gamma$.

$\mathrm{C}-\mathrm{X}-\mathrm{C}$ chemokines induce neutrophil, but not monocyte, migration, whereas $\mathrm{C}-\mathrm{C}$ chemokines act predominantly on monocytes and macrophages with no activity on neutrophils $(22,23,25,26)$. Both the C-X-C and C-C family members act on a number of cell types, including basophils, eosinophils, lymphocytes, endothelial cells, epithelial cells, and SMCs. They have also been shown to induce human leukocyte migration both in vitro and in vivo $(25,26,29-32)$. Lalani et al. (46) have tested the ability of M-T7 to bind to a variety of human and mouse chemokines using a gel-shift assay. It was found that M$\mathrm{T} 7$ binds to all members of the C-C subfamily (RANTES, MIP-1 $\alpha$, MCP-1, MCP-3), the C-X-C subfamily (IL-8, platelet factor 4 ), and the $\mathrm{C}$ subfamily (mouse lymphotaxin) (45). In sections taken at early times after angioplasty in the rat angioplasty injury study (0 hour, 4 hours, 24 hours) M-T7 inhibited invasion of macrophages, but not $\mathrm{T}$ lymphocytes nor smooth muscle cells. This early inhibitory activity is consistent with an inhibition of chemokine-directed cellular migration, but may be due to either direct chemokine binding or to altered regulation of chemokine expression as noted above.

The rat model of vascular balloon injury is characterized by a predominant smooth muscle cellular response with less-pronounced fatty plaque areas or macrophage/foam cell invasion at sites of damage. The cholesterol-fed rabbit model of balloon injury has a more pronounced inflammatory cell component with areas of lipid-filled macrophage invasion. In both cases, however, significant reductions in plaque area were detected with M-T7 infusion. Based upon previous work demonstrating a reduction in plaque growth with IFN treatment in cholesterol-fed rabbits (19), the fact that M-T7 protein known to bind IFN- $\gamma$ and chemokines reduced plaque also suggests a predominant effect from chemokine binding in the inhibition of plaque in this rabbit model of balloon injury. This also suggests that M-T7 is acting at an early initiating step in atherogenesis and that this step is common to both smooth muscle and macrophage cellular responses and invasion and later proliferation at sites of vessel wound healing. These findings also support chemokine activity as a central mediator in the initiation of plaque growth after angioplasty.

In conclusion, we have demonstrated that M-T7, an IFN $\gamma$-R homologue and chemokine-binding protein, inhibits plaque growth and inflammatory cell invasion into the vascular wall after balloon injury in both rat and rabbit models. Our study suggests that chemokine activity plays a greater role than IFN- $\gamma$ activity in early atherogenesis after vascular injury.

\section{Acknowledgments}

This research was supported in part by grants from the Heart and Stroke Foundation of Canada, the Medical Research Council of Canada, the John P. Robarts' Research Institute, and Viron Therapeutics Inc.

\footnotetext{
1. Libby, P., and Clinton, S.K. 1992. Cytokines as mediators of vascular pathology. Nowv. Rev. Fr. Hematol. 34(Suppl.):S47-S53.

2. Hansson, G.K. 1994. Immunological control mechanisms in plaque formation. Basic Res. Cardiol. 89(Suppl. 1):41-46.

3. Terkeltaub, R., Boisvert, W.A., and Curtiss, L.K. 1998. Chemokines and atherosclerosis. Curr. Opin. Lipidol. 9:397-405.
} 
4. Wang, J.M., Shen, W., and Su, S. 1998. Chemokines and their roles in cardiovascular diseases. Trends Cardiovasc. Med. 8:169-174.

5. Wang, N., et al. 1996. Interleukin 8 is induced by cholesterol loading of macrophages and expressed by macrophage foam cells in human atheroma. J. Biol. Chem. 271:8837-8842.

6. Neilken, N.A., Coughlin, S.R., Gordon, D., and Wilcox, J.N. 1991. Monocyte chemoattractant protein-1 in human atheromatous plaques. J. Clin. Invest. 88:1121-1127.

7. Navab, M., et al. 1991. Monocyte transmigration induced by modification of low density lipoprotein in cocultures of human aortic wall cells is due to induction of monocyte chemotactic protein 1 synthesis and is abolished by high density lipoprotein. J. Clin. Invest. 88:2039-2046.

8. Sica, A., et al. 1990. Monocyte chemotactic and activating factor gene expression induced in endothelial cells by IL-1 and tumor necrosis factor. J. Immunol. 144:3034-3038.

9. Reape, T.J., et al. 1999. Expression and cellular localization of the CC chemokines PARC and ELC in human atherosclerotic plaques. Am. J. Pathol. 154:365-374.

10. Ramshaw, A.L., Roskell, D.E., and Parums, D.V. 1994. Cytokine gene expression in aortic adventitial inflammation associated with advanced atherosclerosis. J. Clin. Pathol. 47:721-727.

11. Wysocki, S.J., et al. 1996. Monocyte chemoattractant protein-1 gene expression in injured pig artery coincides with early appearance of infiltrating monocyte/macrophages. J. Cell. Biochem. 62:303-313.

12. Szekanecz, Z., Shah, M.R., Pearce, W.H., and Koch, A.E. 1994. Human atherosclerotic abdominal aortic aneurysms produce interleukin (IL)-6 and interferon-gamma but not IL-2 and IL-4: the possible role for IL-6 and interferon gamma in vascular inflammation. Agents Actions. 42:159-162.

13. Sen, G.C. 1997. The interferons. In Cytokines in health and disease. 2 nd edition. D.G. Remick and J.S. Friedland, editors. Marcel Dekker Inc. New York, New York, USA, Basel, Switzerland, and Hong Kong. 99-208.

14. Vaddi, K., Nicolini, F.A., Mehta, P., and Mehta, J.L. 1994. Increased secretion of tumor necrosis factor- $\alpha$ and Interferon- $\gamma$ by mononuclear leukocytes in patients with ischemic heart disease. Circulation. 90:694-699.

15. Stopeck, A.T., Vahedian, M., and Williams, S.K. 1997. Transfer and expression of the interferon gamma gene in human endothelial cells inhibits vascular smooth muscle cell growth in vitro. Cell Transplant. 6:1-8.

16. Jonasson, L., Hansson, G.K., Bondjers, G., Noe, L., and Etienne, J. 1990. Interferon-gamma inhibits lipoprotein lipase in human monocyte derived macropghages. Biochim. Biophys. Acta. 1053:43-48.

17. Geng, Y.J., and Hansson, G.K. 1992. Interferon- $\gamma$ inhibits scavenge receptor expression and foam cell formation in human monocytederived macrophages. J. Clin. Invest. 89:1322-1330.

18. Suzuki, H., et al. 1989. Interferon- $\gamma$ modulates messenger RNA levels of c-sis (PDGF-B chain), PDGF-A chain, and IL-1 $\beta$ genes in vascular endothelial cells. Am. J. Pathol. 134:35-42.

19. Wilson, A.C., Schaub, R.G., Goldstein, R.C., and Kuo, P.T. 1990. Suppression of aortic atherosclerosis in cholesterol-fed rabbits by purified rabbit interferon. Arteriosclerosis. 10:208-214.

20. Kuo, P.T., Wilson, A.C., Goldstein, R.C., and Schaub, R.G. 1984. Suppression of experimental atherosclerosis in rabbits by interferon-inducing agents. J. Am. Coll. Cardiol. 3:129-134.

21. Russell, P.S., Chase, C.M., Winn, H.J., and Colvin, R.B. 1994. Coronary atherosclerosis in transplanted mouse hearts. III. Effects of recipient treatment with a monoclonal antibody to interferon- $\gamma$. Transplantation. $\mathbf{5 7 : 1 3 6 7 - 1 3 7 1 .}$

22. Tellides, G., et al. 2000. Interferon- $\gamma$ elicits arteriosclerosis in the absence of leukocytes. Nature. 403:207-211.

23. Taub, D.D. 1996. Chemokine-leukocyte interactions. The voodoo that they do so well. Cytokine Growth Factor Rev. 7:355-376.

24. Terkeltaub, R., Boisvert, W.A., and Curtiss, L.K. 1998. Chemokines and atherosclerosis. Curr. Opin. Lipidol. 9:397-405.

25. Gerszten, R.E., et al. 1999. MCP-1 and IL-8 trigger firm adhesion of monocytes to vascular endothelium under flow conditions. Nature. 398:718-723.

26. Schall, T.J., and Bacon, K.B. 1994. Chemokines, leukocyte trafficking, and inflammation. Curr. Opin. Immunol. 6:865-873.

27. Ben-Baruch, A., Michiel, D.F., and Oppenheim, J.J. 1995. Signals and receptors involved in recruitment of inflammatory cells. J. Biol. Chem. 270:11703-11706.

28. Gosling, J., et al. 1999. MCP-1 deficiency reduces susceptibility to atherosclerosis in mice that overexpress human apolipoprotein B. J. Clin. Invest. 103:773-778.

29. Boring, L., Gosling, J., Cleary, M., and Charo, I.F. 1998. Decreased lesion formation in CCR $2^{-/}$mice reveals a role for chemokines in the initiation of atherosclerosis. Nature. 394:894-897.

30. Boring, L., et al. 1997. Impaired monocyte migration and reduced type 1 (Th1) cytokine responses in C-C chemokine receptor 2 knockout mice. J. Clin. Invest. 100:2552-2561.

31. Boisvert, W.A., Santiago, R., Curtiss, L.K., and Terkeltaub, R.A. 1998. A leukocyte homologue of the IL-8 receptor CXCR-2 mediates the accumulation of macrophages in atherosclerotic lesions of LDL receptordeficient mice. J. Clin. Invest. 101:353-363.

32. Fairchild, R.L., Van Buskirk, A.M., Kondo, T., Wakely, M.E., and Orosz, C.G. 1997. Expression of chemokine genes during rejection and long term acceptance of cardiac allografts. Transplantation. 63:1807-1812.

33. Furukawa, Y., et al. 1999. Anti-monocyte chemoattractant protein$1 /$ monocyte chemoattractant and activating factor antibody inhibits neointimal hyperplasia in injured rat carotid arteries. Circ. Res. 84:306-314

34. Tanaka, H., et al. 1993. Sustained activation of vascular cells and leukocytes in the rabbit aorta after balloon injury. Circulation. 88:1788-1803.

35. Reidy, M.A., Fingerle, J., and Lindner, V. 1992. Factors controlling the development of arterial lesions after injury. Circulation. 86(Suppl):III43-III46.

36. Swartz, R.S., Holmes, D.R., and Topol, E.J. 1992. The restenosis paradigm revisited: an alternative proposal for cellular mechanisms. J. Am. Coll. Cardiol. 20:1284-1293.

37. Mintz, G.S., et al. 1998. Treatment of in-stent restenosis. Semin. Interv. Cardiol. 3:117-121.

38. Topol, E.J., et al. 1993. A comparison of directional atherectomy with coronary angioplasty in patients with coronary artery disease. $N$. Engl. J. Med. 329:221-227.

39. Ross, R. 1993. Rous-Whipple Award Lecture. Atherosclerosis: a defense mechanism gone awry. Am. J. Pathol. 143:987-1002.

40. Dzau, V.J., Gibbons, G.H., Cooke, J.P., and Omoigui, N. 1993. Vascular biology and medicine in the 1990s: scope, concepts, potentials, and perspectives. Circulation. 87:705-719.

41. McFadden, G., et al. 1995. Interruption of cytokine networks by poxviruses: lessons from myxoma virus. J. Lenkoc. Biol. 57:731-738.

42. Nash, P., et al. 1999. Immunomodulation by viruses: the myxoma story. Immunol. Rev. 168:103-120.

43. McFadden, G., and Graham, K. 1994. Modulation of cytokine networks by poxvirus: the myxoma virus model. Seminars Virology. 5:421-429.

44. Barry, M., et al. 1997. Virus encoded cytokines and cytokine receptors. Parasitology. 115(Suppl.):S87-S100.

45. Upton, C., Mossman, K., and McFadden, G. 1992. Encoding of the IFNgamma receptor by myxoma virus. Science. 258:1369-1372.

46. Lalani, A.S., and McFadden, G. 1997. Secreted poxvirus chemokine binding proteins. J. Leukoc. Biol. 62:570-576.

47. Lucas, A., et al. 1996. Virus-encoded serine proteinase inhibitor SERP-1 inhibits atherosclerotic plaque development after balloon angioplasty. Circulation. 94:2890-2900.

48. Dai, E., et al. 1997. Calreticulin, a potential vascular regulatory protein, reduces intimal hyperplasia after vascular injury. Arterioscler. Thromb. Vasc. Biol. 17:2359-2368.

49. Miller, L.W., et al. 2000. Inhibition of transplant vasculopathy in a rat aortic allograft model after infusion of an anti-inflammatory viral serpin. Circulation. 101:1598-1605.

50. Mossman, K., and McFadden, G. 1996. Myxoma virus M-T7, a secreted homolog of the interferon gamma receptor, is a critical virulence factor for the development of myxomatosis in European rabbits. Virology. 